\title{
IMP 8 magnetosheath field comparisons with models
}

\author{
Z. Kaymaz \\ Istanbul Technical University, Faculty of Aeronautics and Astronautics, Maslak, 80626, Istanbul, Turkey
}

Received: 1 September 1997 / Revised: 17 November 1997 / Accepted: 19 November 1997

\begin{abstract}
This study presents cross-sectional vector maps of the magnetic field derived from IMP 8 magnetometer in the magnetosheath at $30 \mathrm{R}_{\mathrm{e}}$ behind the Earth. In addition the vector patterns of the magnetosheath field for northward, southward, and east-west interplanetary magnetic field (IMF) directions are qualitatively compared with those obtained from the Spreiter-Stahara gas dynamic (GD) and Fedder-Lyon magnetohydrodynamic models (MHD). The main purpose is to display the cross-sectional differences in relation to the dayside merging with different IMF directions, allowing the reader to make direct visual comparisons of the vector patterns. It is seen that for east-west IMF directions, the data-based and MHD-based patterns differ noticeably in a similar way from the GD model, presumably reflecting the influence of dayside magnetic merging of the Earth's magnetic field with the y-component of the interplanetary magnetic field. All three northward IMF cross sections show comparable field draping patterns as expected for a closed magnetosphere. For southward IMF case, on the other hand, differences between the three cross-sectional patterns are greater as seen in the field vector sizes and directions, especially closer to the magnetopause where more disturbed magnetospheric conditions are known to be exist. The data comparisons with the MHD and GD models show that the differences result from the magnetic field-flow coupling and that the effects of dayside reconnection are present in IMP 8 magnetic field measurements.
\end{abstract}

Keywords. Vector maps · IMP 8 magnetometer · Interplanetary magnetic fields

\section{Introduction}

The magnetosheath, a thermalized and deflected solar wind plasma region between the bow shock and magnetopause, is one of the least studied regions of space. Its magnetic and flow properties have been modeled by using either gas dynamics (GD) or aerodynamic calculations to represent the flow around a blunt object, or magnetohydrodynamic (MHD) simulations which couple the aerodynamic calculations with the electromagnetic equations. The comparisons of the gas dynamic model results with the observational data have shown that in the absence of the magnetic field, the model predicts the flow and field behavior in the magnetosheath fairly well (e.g., Zhang et al. 1996). However, MHD model results are expected to be in closer agreement with the observations owing to the coupling between the magnetic field and flow. My purpose is to compare the cross-sectional vector patterns of the magnetosheath field from IMP 8 data, a gas dynamic model and MHD models. First there is a description how these two models differ from or resemble the magnetosheath data of IMP 8, and then the comparisons are discussed in terms of the dayside merging signatures. This study differs from earlier works in two ways: first, as summarized in the next section, almost all of the previous work was done in the forward part of the magnetosheath. Here, the magnetosheath is studied behind the terminator at $-30 \mathrm{Re}$ where magnetic forces have had longer to act on the plasma. Second, this is the first time an MHD model (Fedder et al., 1995; Fedder and Lyon, 1995) is tested against the observational data in the nightside magnetosheath. Brief descriptions of the IMP 8 data and models used for the comparisons follow.

\subsection{Imp 8 magnetometer data in the magnetosheath}

The observations used here are described in detail in Kaymaz et al. (1992). Briefly, the 5-minute averaged magnetometer data from IMP 8 for 1978-1982 were obtained from the data archive at the NSSDC. After separation of the magnetotail data from the $\mathrm{x}=-25$ to $-31 R_{\mathrm{e}}$ interval, these data were sorted into four subsets (northward, southward, eastward and westward) ac- 
cording to the prevailing IMF clock angle as observed on ISEE 3 (with adjustment for the solar wind advection time). The IMP 8 positions and the field vectors were rotated to a common IMF direction in the center of each $45^{\circ}$ clock angle sector. Also the IMP 8 data corresponding to the solar-magnetic toward sectors were converted into solar-magnetic away sectors by changing the field vectors' directions and moving the spacecraft appropriately, thus all data are as if obtained during away sectors. The last correction is made for the tail field flaring and then the data are "smoothed" in the cross-sectional (GSM- $y z$ ) plane by averaging the vectors within a $4 R_{\mathrm{e}}$ radius of each vector. The results are cross-sectional maps of the magnetosheath field for northward, southward and east-west IMFs as shown in the top row of Fig. 1. Our analysis here focuses on these cross-flow components of the magnetosheath magnetic field only.

Kaymaz et al. (1992) discussed the attributes of observed magnetosheath field draping patterns in terms of their departures from symmetry with respect to the IMF direction. They pointed out that the draping appears most symmetric for northward and southward cases, while the equatorial IMF case is distorted by a twisted field draping pattern in the magnetosheath close to the magnetopause. This twisting appears as a rotation of the bifurcation points, i.e., the points where the last draped field line at the magnetopause splits to go around the body of the tail, away from the axis of symmetry as determined by the IMF direction. They further subdivided the data into smaller IMF clock angle sector bins to determine how much the line through the bifurcation points rotates with respect to the IMF direction. These results will be compared with the models later. Of principal interest here is the suggestion that the cause of the observed rotation of the bifurcation points is reconnection at the dayside magnetopause.

\subsection{About the gas-dynamic model}

The flow properties of the solar wind in the magnetosheath as it moves around the Earth's magnetosphere can be modeled reasonably well by means of gas dynamic calculations, neglecting the magnetic field effects and treating the magnetopause as an impenetrable obstacle. These gas dynamic models of the solar wind flow have provided the theoretical basis for several decades for understanding and interpreting the phenomena occurring in space around terrestrial bodies. The general value and usefulness of the results based on these fluid models are well established, and have advanced to the point where theoretical calculations are now used to predict important planetary-solar wind interaction characteristics such as the bow shock position (Slavin et al. 1983). The fundamental assumption of gas dynamic models is that the average bulk properties of the solar wind flow around a planetary magneto/ionosphere can be adequately described by the continuum equations of magnetohydrodynamics for a single-component ideal gas with electrical conductivity, zero viscosity and thermal conductivity. In the magne- tohydrodynamic equations, the terms including the magnetic field are small almost everywhere, compared to the flow terms in the solar wind. Therefore, as an approximation, the hydrodynamic and electromagnetic equations can be decoupled. The hydrodynamic equations are first solved for the flow field around an impenetrable blunt obstacle; then, with the flow properties known, the steady state interplanetary magnetic field is determined by assuming a frozen-in field. Two other features of the model are that it uses a Newtonian pressure formula $\left(P_{N}=P_{s t} \operatorname{Cos}^{2}(\Psi)\right)$, to calculate the magnetopause boundary shape in an independent step and that it employs an axisymmetric approximation for the magneto/ionopause shape. The description of the model's physics and the computations has been given in detail by Spreiter et al. (1966, 1968), Spreiter and Alksne (1969), Stahara et al. (1979) and Spreiter and Stahara (1980).

The gas dynamic model of the magnetosheath has been applied to both the magnetized and unmagnetized planets (Spreiter et al. 1966; Behannon and Fairfield 1969; Spreiter and Alksne 1970; Fairfield 1976; Spreiter and Stahara 1980; Luhmann et al. 1984, 1986; Zhang et al. 1996). In particular, using IMP 1 and IMP 2 spacecraft observations Fairfield (1967) found that the terrestrial data behaved, at least qualitatively, as predicted by the model. For example, the measured magnetosheath magnetic field, in some regions, behaved like IMF convected through the bow shock which increases its magnitude but changes its orientation relatively little. Moreover, as the field convects deeper into the magnetosheath, it changes direction until it lies approximately tangent to the magnetopause. In another example, Behannon and Fairfield (1969) showed that the expected draping pattern of the IMF is present in the nightside magnetosheath field. Compared to the simultaneous interplanetary field, the draped field varies from greater than four near the stagnation point to values less than unity in the downstream magnetosheath, in overall agreement with the gas dynamic model results.

However, even though the GD model is found to be adequate for predicting the observed properties of the magnetosheath field and flow on large scales, the lack of magnetic coupling in the gas dynamic equations cause the model to fail in regions close to the magnetopause. Because the magnetic forces become dominant in the inner dayside magnetosheath and because the geomagnetic field interconnects with the IMF and violates the gas dynamic model assumption of an impenetrable magnetopause boundary, magnetic reconnection particularly affects the field draping geometry at the magnetopause as shown in Kaymaz et al. (1992) for IMFs in the equatorial plane. Further, the field structure is different qualitatively and quantitatively for northward and southward IMFs. Proper representation of conditions at the magnetopause requires that the magnetic field and flow be solved together, with the inclusion of the IMF orientation and the coupling with the magnetospheric fields. This coupling is also important for tail geometry and dynamics (Kaymaz et al., 1994, 1995). 
The density and field variations along the stagnation line in the dayside magnetosheath were investigated by Zwan and Wolf (1976) and recently by Song et al. (1992). Zwan and Wolf (1976) showed that as a result of the compressed magnetic field tubes, the magnetic field strength increases while the density decreases near the nose of the magnetopause. In their study, Song et al. (1992) observed that while the density stays relatively constant over most of the magnetosheath, it dramatically increases prior to a decrease seen before the actual subsolar magnetopause crossing. Both results do not agree with the gas dynamic model which shows a monotonic density increase along the stagnation line. Song et al. (1992) also showed that the observed density increase is anticorrelated with the magnetic field strength and associated with a slow mode wave. They interpret that this slow mode transition can effect the final field and flow patterns that diverge around the magnetopause. These studies demonstrate that, for a more realistic representation of the magnetosheath flow and field, both the magnetic reconnection effects at the magnetopause and the effects caused by the changes in the plasma density must be taken into account.

The design of the Spreiter-Stahara (1980) gas dynamic magnetosheath model used in this study allows easy comparison with the data. As inputs, the code requires the obstacle shape, free stream Mach number, and the ratio of specific heats. (See Luhmann et al., 1986 for another application of this code). For the comparisons here, an obstacle shape factor of 0.25 , which determines the extent of magnetopause flaring, a free stream Mach number (magnetosonic) of 4.5, and value of $5 / 3$ for the specific heat ratio were adopted. The model results are quite insensitive to moderate increases in Mach number, while the chosen obstacle resembles the documented magnetopause shape (e.g., Petrinec and Russell, 1933). The code also requires the IMF as input. Given these inputs, the code first computes a hydrodynamic flow field and stores it in a file. This flow field is then used to compute the frozen-in convected magnetic field for the specified IMF. The output consists of magnetic field components calculated for the fixed flow field and IMF at the spatial positions of the observed vectors. The magnetic field has no effect on the flow in this model, and is everywhere tangent to the magnetopause at the inner boundary.

\subsection{About the MHD model}

Model of the magnetosheath flow which take the magnetic forces into account, i.e., MHD models, are not yet developed to the point of being easily used as the simpler gas dynamic model already is. The treatment of the dissipative terms in the MHD model requires finer spatial grids over extended regions. Even the most sophisticated global MHD models have poor spatial resolution and are costly to run. However, improving computer capabilities are enabling the MHD modelers to refine their techniques so that we are proceeding toward more realistic simulations of the Earth's coupled magnetosphere and magnetosheath.
The MHD model used here is based on numerical solutions of the ideal MHD equations applied to the solar wind interaction with a dipole field. This model includes an ionospheric inner boundary with realistic conductivities and an outflow boundary condition at the back of the simulation box. By its nature, it also includes diffusion which affects the solutions. In this case, there are no diffusive and viscous terms added in the equations that are effective on length scales approaching the ion gyro-radius and on temporal scales approaching the inverse of the ion gyro frequency. The smallest time scale is a fraction of a second. At these scales, neither viscosity nor diffusive effects are important. Thus, the numerical calculation gives the geometrical evolution of the model fields resulting from their interconnection at the magnetopause. A detailed description of the model attributes is given by Fedder et al. (1995) and Fedder and Lyon (1995). In the code used here, the solar wind is in all cases assumed to be steady with density $\rho=1.125$ $\times 10^{-23} \mathrm{~g} / \mathrm{cm}^{3}\left(\mathrm{n} \sim 5.0 \mathrm{~cm}^{-3}\right)$, velocity, $V_{S W}=400 \mathrm{~km} /$ sec, (magnetosonic) Mach number, $M=7$, and IMF magnitude $|B|=5 \mathrm{nT}$. In the x-direction (the Earth-Sun axis coordinate), the outer boundaries of the model are located at $\mathrm{x}=+25 \mathrm{R}_{\mathrm{e}}$ and $-100 \mathrm{R}_{\mathrm{e}}$. In this work, the results for the magnetic field only for model runs with steady duskward ( $+B_{y}$ GSM), northward ( $+B_{Z}$ GSM) and southward $\left(-B_{z}\right.$ GSM) IMFs are shown.

In their paper, Fedder et al. (1995) present the topology of their global MHD model fields in the magnetosheath close to the magnetopause for different IMF directions. Their results could be understood in terms of dayside reconnection. They also showed that the angle between the IMF direction and the separatrix in the magnetosheath where two topologically different fields (from the magnetosphere and from the magnetosheath) meet increases as the IMF changes its orientation from north to south. These results agrees well with the results of Kaymaz et al. (1992) and encourage us to make the comparisons given in this work. We give here the first comprehensive comparison between magnetosheath data and Fedder-Lyon MHD model. This study is complimentary to an earlier comparison between magnetotail data and this MHD model (Kaymaz et al., 1995).

Comparisons of magnetosheath data with MHD model results and gas dynamic model results are made using cross sections of the tail's magnetosheath. The accuracy of the gas dynamic magnetosheath model is examined, as is the magnetosheath part of the global MHD model. The IMP 8 data set gives fairly complete cross-sectional patterns of the magnetic field in an annular ring near $x=-30 R_{\mathrm{e}}$. The comparisons made here for three different IMF directions (northward, southward, east-west) show the effects of dayside reconnection on the magnetosheath field.

\section{Comparisons and discussion}

In this section, we present the results from the field vector maps for each IMF clock angle sector (north- 
ward, southward, and east-west) and discuss the features revealed in these maps in terms of the dayside magnetic reconnection effects.

\subsection{Northward IMF}

Figure 1 shows $y z$-plane projections of the sheath magnetic fields for northward, southward, and eastwest IMFs derived from IMP 8 data, GD and MHD models. In all panels, the sheath field vectors are normalized to the upstream field values to make comparisons. The panels $(\mathrm{a}, \mathrm{b}, \mathrm{c})$ in the top row represent the IMP data. As seen in Fig. 1a, the northward IMF shows a symmetric draping around the magnetopause. The fields become smaller where the fields split to divert around the magnetopause and where they rejoin on the other side. We call the locations where the field vectors start diverging and converging the bifurcation points, and the line that connects them the bifurcation line (see Fig. 5b). The bifurcation line here is parallel to the IMF direction, northward in this panel, as one expects in the absence of magnetic merging on the dayside magnetopause for northward IMFs.

Figure 1d gives the gas dynamic model vectors calculated at the same positions as in the top panel. The IMF used to obtain the model fields is $\operatorname{IMF}\left(B_{x}, B_{y}\right.$, $\left.B_{z}\right)=(-3.6,0,5.7) \mathrm{nT}$, which is the same total magnitude as the observed fields Fig. 1a. The model field vectors were normalized to the magnitude of the IMF and smoothed in the same way as the IMP 8 vectors (i.e., $4 R_{e}$ circle averaging at each vector location). Qualitatively, the magnetic field draping pattern produced by the gas dynamic model for northward IMFs agrees with the observed field pattern. The field draping in both cases is symmetric with respect to the IMF direction. The agreement in this case between the data-based vector pattern and the GD model vector pattern indicates that boundary processes for northward IMF (i.e., magnetopause permeability and tangential stress) do not substantially affect the draping of magnetosheath fields as seen in the $y z$-plane at $-30 \mathrm{R}_{\mathrm{e}}$ down tail distance. Qualitatively at least, in the absence of dayside merging, as expected during northward IMF, the magnetosheath field around the magnetosphere appears symmetrical, undisturbed, and can be approximated by the gas dynamic model.

In spite of the SC agreements, differences arise in the field magnitudes as illustrated in Fig. 2a. Figure 2a is a vector-by-vector subtraction of Fig. 1d from Fig. 1a. These vectors quantify the difference between the model and observed field magnitudes. The difference vectors tend to point down, which means that, on average, model fields exceed the observed fields. We can determine the amount by which the model fields differ from the observed fields by finding the average difference field in the $z$-direction, that is $B_{z}$. It is found to be about $22 \%$ of the upstream field magnitude. This means that compared to the interplanetary magnetic field, the GD model over-estimates the $B_{z}$ fields in the magnetosheath by $22 \%$ on the average. This corresponds to $1.48 \mathrm{nT}$ for an interplanetary field magnitude of $6.74 \mathrm{nT}$ in this panel.

The subsolar magnetosheath field is subject to compression at the bow shock (Fairfield, 1967) that increases the field magnitude to up to four times its upstream value (depending on shock normal) (Crooker et al., 1982). However, there is also a more general compression at the nose of the obstacle due to the density depletion in the diverging magnetosheath flow (Zwan and Wolf, 1976). In Fig. 2a, the field magnitudes predicted by the model are clearly larger than those observed near the magnetosheath inner boundary, especially where draping is strongest. This is also the region where the gas dynamic model fails to represent the real conditions because of neglected effects of the magnetic pile-up at the magnetopause (Alksne, 1967).

Figure 1g shows the MHD model vectors. The similarities between the MHD model and both IMP 8 data and the GD model vectors seen in this panel are striking. This agreement shows that the northward interaction produces the most idealized magnetosheath field configuration. The field vectors in the outer magnetosheath of the MHD model are larger than their GD counterparts, and both model vectors are larger than the observed fields. However, in the inner regions, the MHD field vectors are in closer agreement with the data than the GD model vectors. They also agree better with the data on the dawn and dusk inner boundary regions where the fields run tangential to the boundary, while the GD model vectors there are too large. Especially at northern bifurcations locations, the MHD model field resembles the observed field more than the GD model. The MHD vectors differ from the data, however, at the innermost southern bifurcation locations, where they are in the opposite directions to those observed. Since the global MHD model includes the coupled magnetosphere and magnetosheath, with a broadened magnetopause boundary for northward IMF, there remains ambiguity as to where the magnetosheath ends and tail lobe begins. The MHD model vectors at those locations which are seen to be more taillike (outward) than magnetosheath-like (inward) in the cross-sectional plane may result from this ambiguity. This, then, can give rise to larger difference vectors at the southern bifurcation locations very close to the magnetopause in the MHD panel, and obscure the comparisons there.

The difference vectors for the northward IMF case of the MHD model are shown in Fig. 2b. The primary contrasts to the difference field of the GD model (Fig. 2a) are seen in the inner magnetosheath. Overall, the average $B_{z}$ difference fields are about the same magnitude as those in GD difference panel. The difference vectors show that while the GD model is in agreement with the observations throughout most of the magnetosheath, in the inner regions along the flanks and at the bifurcation regions, the MHD model panel provides the better comparison. 

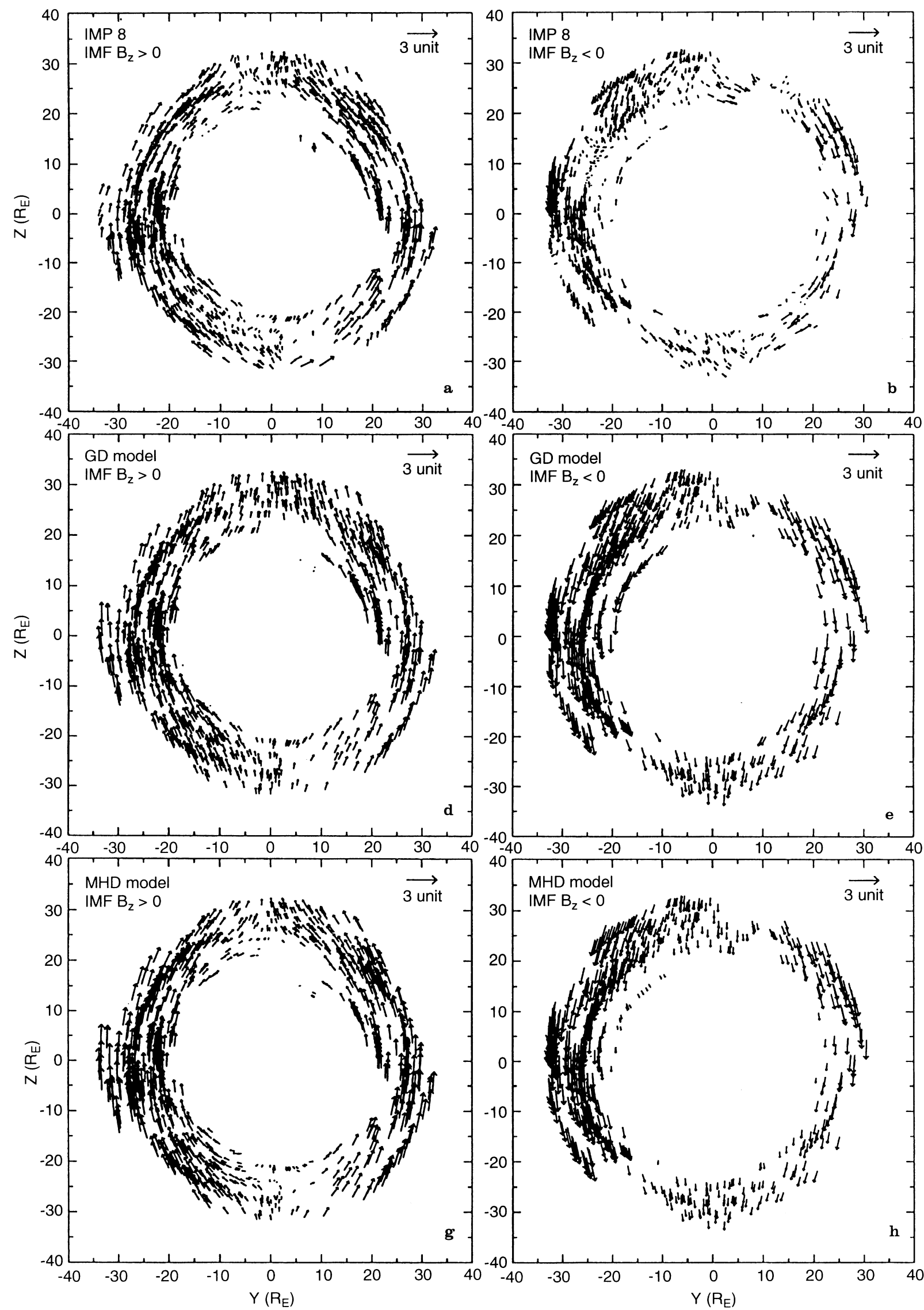

Fig. 1a-i. Upper panels: IMP 8 magnetic field vectors in the yz-plane at $-30 \mathrm{Re}$ for a northward, b southward, and $\mathbf{c}$ equatorial IMFs. Middle Panels: gas dynamic model vectors for $\mathbf{d}$ northward, e southward, and f equatorial IMFs. Lower panels: MHD model vectors for $\mathbf{g}$ northward, h southward, $\mathbf{i}$ equatorial IMFs. The sheath vectors are normalized to upstream field values 

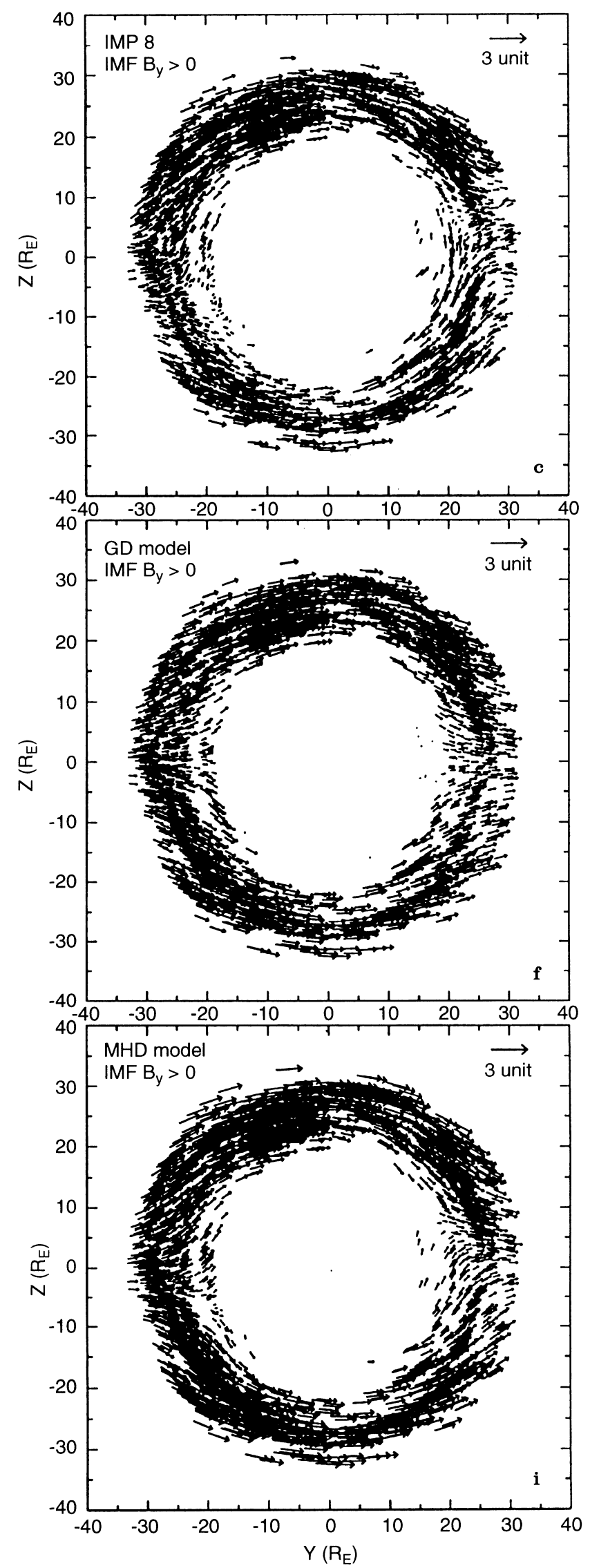

Fig. $1 \mathrm{c}, \mathrm{f}, \mathrm{i}$

\subsection{Southward IMF}

Figure $1 \mathrm{~b}$ shows the IMP 8 field vectors in the magnetosheath for southward IMF pattern. There are several distinctive features in this southward IMF pattern compared to the northward IMF pattern. The first is the direction and size of the field vectors and their apparent irregular, turbulent structure. The second is the localization of the most irregular fields on the northern dawn and southern dusk sides of the magnetopause. The southward IMF field vectors, especially along the inner annulus of the cross section, are much smaller than their counterparts in the northward IMF panel. Irregularities in the fields break the smooth draping of the magnetic field in the northward IMF panel. These irregular vectors may be thought of as indicating a disturbed magnetosphere. The preferred appearance of these irregular vectors on the northern dawn and southern dusk sides of the magnetopause are consistent with the idea that boundary interactions may be taking place. These regions could be the "debris" from the interconnection between the interplanetary field and dipole field at the dayside magnetopause.

Figure 1e shows the gas dynamic model vectors for the southward IMF case corresponding to Fig. 1b. The IMF components used in the model are $B_{x}=-4 \mathrm{nT}$, $B_{y}=0, B_{z}=-6.69 \mathrm{nT}$. It is clear that the northward and southward IMF panels of the GD model show almost the same draping pattern, as expected in the absence of reconnection effects. The only difference between Fig. 1d and Fig. 1e is the magnitude of the fields which are controlled by the solar wind parameters in the model. Comparing Fig. 1e of the GD model with Fig. $1 b$ of the data shows that the difference is greater here than in the northward IMF case. As noted, the observed draping pattern shows irregularities at certain places, while the southward panel of the GD model shows smooth draping around the object. The irregular character and sizes of the vectors in this plane, not seen for the northward IMF case, suggests the presence of boundary interactions for the southward IMF.

These anomalous, irregular vectors seen in the observational data (Fig. 1b) could be due either to the accidental features of the boundary occurring during southward IMF, or due to the boundary processes in the presence of a finite IMF $B_{y}$ present in the southward IMF data subset. While in the first case, we might be observing a transient phenomenon, in the later case, we might be seeing the residual signatures of the interconnected fields at the diagonal corners of the draping pattern resulting from the non-zero IMF $\mathrm{B}_{\mathrm{y}}$ of about 2 $\mathrm{nT}$. To determine if these anomalous vectors are just an accidental feature of the spacecraft trajectory and a unique transient phenomena occurring during the southward IMF, each individual anomalous field vector seen on the northern dawn and southern dusk regions was inspected. This closer inspection shows that the anomalies come from more than one orbit, thereby implying they could be persistent. The irregular directions and sizes of the vectors in the $y z$-plane, not seen in 

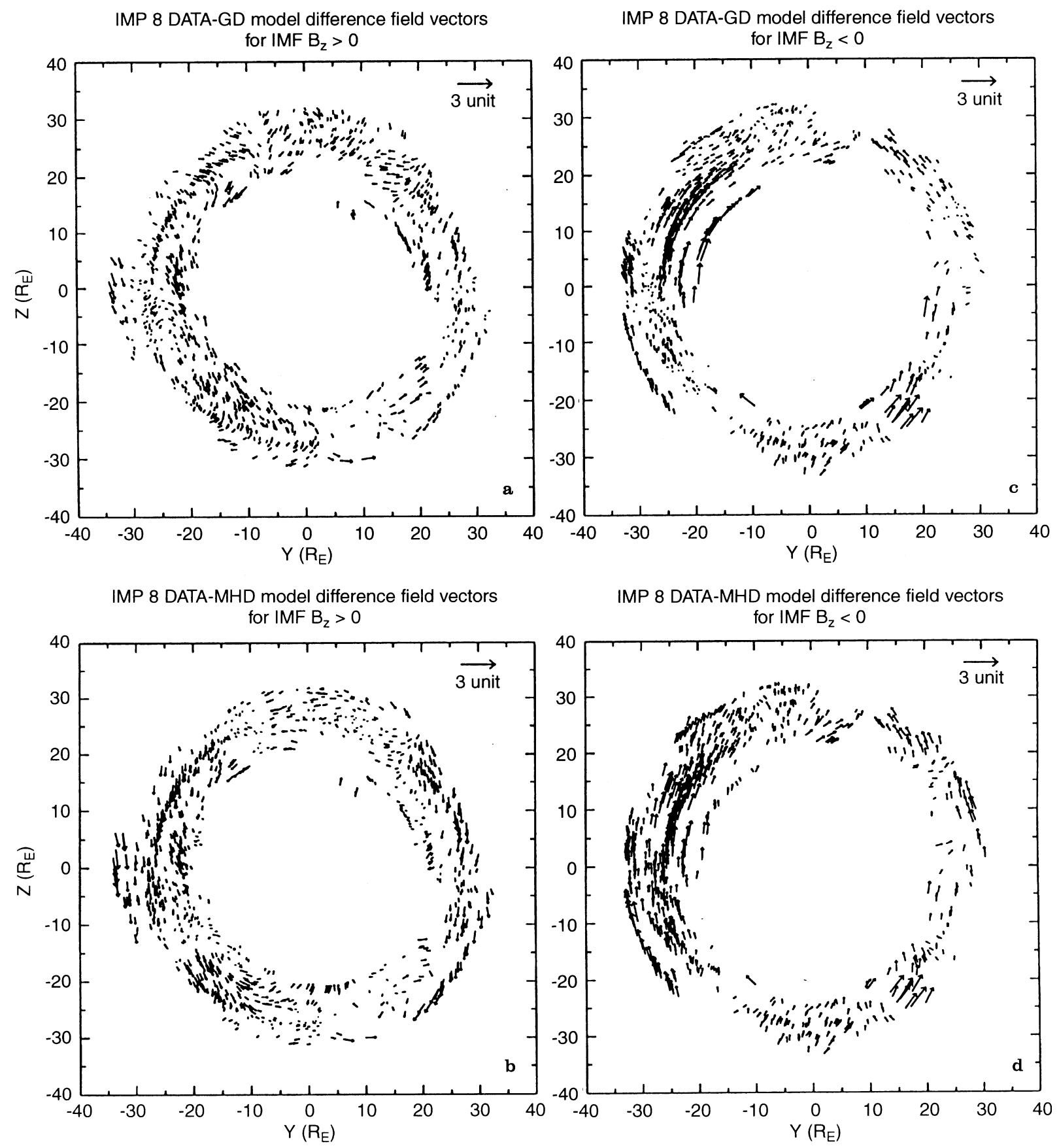

Fig. 2a-d. Upper panels: IMP 8 and gas dynamic model difference vectors for a northward IMFs, b southward IMF difference vectors after $22 \%$ reduction as described in the text. Lower panels: IMP 8 and MHD model difference vectors for $\mathbf{c}$ northward and for d southward IMF

the northward IMF case, must be characteristic of the boundary interactions in the southward IMF case.

To quantify the difference seen in the southward IMF panels of the GD model and the data, the average difference amount of $22 \%$ found for the northward IMF is subtracted from the southward IMF panel of the GD model to determine how much the southward IMF case is different from the hydrodynamical representation. Figure $2 \mathrm{c}$ shows the difference vectors obtained by subtracting Fig. 1e after the $22 \%$ adjustment from Fig. 1b. It is seen that the strongest difference vectors are concentrated at the diagonal corners where the anomalous deviations occur. Thus, Fig. 2c both locates the anomalies and gives their values.

As a conceptual model that is useful for thinking about the geometry of draping expected from boundary interactions, we consider the rotational discontinuity model of the reconnecting or open magnetosphere given by Siscoe and Sanchez (1987). It predicts the locations of the "windows" through which the IMF interconnects with the magnetospheric field. Figure 3 reproduced from Siscoe and Sanchez (1987) shows an open magnetosphere for purely southward (Fig. 3a) and southward IMF with a non-zero $B_{y}$ component (Fig. $3 b$ ). In the 

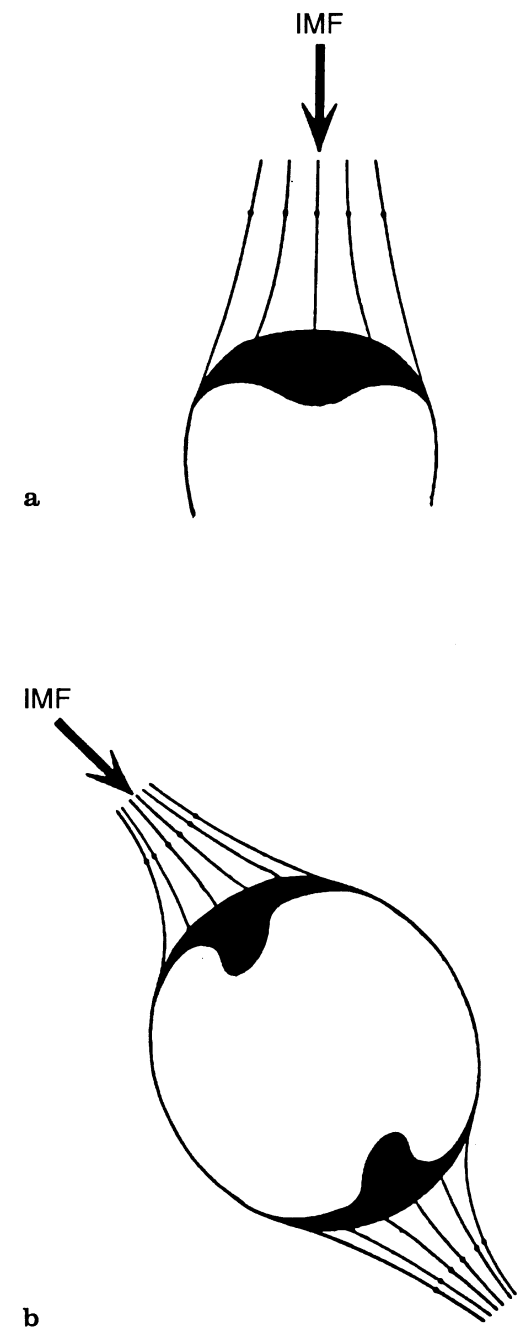

Fig. 3a, b. A phenomenological model of the open magnetosphere from Siscoe and Sanchez (1987) showing magnetospheric "windows", where the magnetosheath field lines interconnect with the geomagnetic field lines, for a southward IMF and $\mathbf{b}$ southward IMF with a non-zero $B_{y}$ component

case of pure southward IMF (Fig. 3a), field lines penetrate into the tail lobes through the plasma mantle (shaded region). The boundary segments at which the fields interconnect determine the "windows". The locations of these windows change with the direction of IMF. In the presence of IMF $B_{y}$ (Fig. 3b), the windows are shifted counterclockwise from the north-south polar locations. The anomalous regions seen in Fig. $2 \mathrm{c}$ are located where one would expect to find magnetosheath field draping affected by these windows into the magnetosphere. In other words, they may correspond to the regions where the magnetotail magnetic field lines are connected to the interplanetary fields in the magnetosheath.

If we assume that the difference fields in Fig. 2c area are a direct measure of the interconnected field, we may use them to estimate the cross-tail electric potential across the magnetosphere applied by the solar wind through these windows. The averaged potential can be found by using the tangential difference field vectors from the relation $\left(\Phi=\left(V_{\text {sheath }}\right) \times\left(B_{\tan }\right) \times(\Delta r)\right)$, where $V_{\text {sheath }}$ is the magnetosheath velocity, $B_{\tan }$ is the tangential difference field at the window locations and $\Delta r$ is the combined magnetosheath width of both diagonal windows which is about $25 \mathrm{R}_{\mathrm{e}}$. For the average tangential field across $\Delta r$ of about $7 \mathrm{nT}$ in Fig. 2c, and an estimated flow speed at the local magnetopause of $V_{\text {sheath }}=368 \mathrm{~km} / \mathrm{s}$ (determined from GD model), we obtain a potential drop of about $400 \mathrm{kV}$. This value is equal to the largest cross-polar potential ever reported (Gurnett and Frank, 1973; Reiff and Luhmann, 1986; Sanchez and Siscoe, 1990). For a more reasonable potential drop, e.g., $50 \mathrm{kV}$, the average tangential field should be only about $1 \mathrm{nT}$. Difference fields of this magnitude cannot be resolved with the technique used here. Thus we conclude that the magnetosheath anomalies seen for southward IMF with a non-zero $B_{y}$ component do not directly measure the amount of connected flux. Nonetheless, they may be persistent distortion features existing near the open tail windows. This conclusion is now being tested with the MHD model that includes the magnetic reconnection at the magnetopause boundary.

Figure $1 \mathrm{~h}$ presents the MHD model vectors for southward IMF. Although the MHD model vectors appear to be similar to their GD model counter-parts, a closer look shows they are smaller than the GD model vectors, especially close to the boundary regions. At the observed anomaly regions, the MHD model gives a better prediction in terms of the field directions and strengths. As a result of the impenetrable inner boundary, the GD model vectors have large tangential components. The MHD model vectors have smaller tangential components and vectors directed inward toward the magnetopause, especially at the northern dawn and southern dusk window regions. This is better seen in the MHD model IMP 8 difference vectors shown in Fig. 2d. Close to the magnetopause boundary, particularly near the anomaly regions, the difference vectors are smaller than those in Fig. 2c. This suggests that even though neither model is precisely like the observational data, the MHD model seems to better approximate the data for southward IMF than does the gas dynamic model. This result supports the inference that the anomalies are signatures of reconnection at the boundary.

\subsection{East-west IMF}

Figure 1c shows the IMP 8 magnetic field vectors in the magnetosheath in the cross-sectional plane for east-west IMF directions. This figure is discussed in Kaymaz et al. (1992) in detail but the highlights from the study are given here as well in order to compare the pattern with that of models. Kaymaz et al. (1992) show that the interconnection between the geomagnetic field and the IMF for different IMF orientations creates very different field topologies near the magnetopause. The effects of reconnection appears as a rotation of the magnetic field pattern at the magnetopause boundary relative to 
the IMF direction. This rotation of the field draping pattern determined from the bifurcation points increases as the IMF angle relative to the Earth's dipole deviates from $0^{\circ}$. It is most extreme when the IMF has a component perpendicular to the dipole together with a southward component. The sense of the rotation is consistent with the merging line picture in the presence of a strong $B_{y}$ component of the IMF (Gonzales et al., 1978). The rotation of the pattern with respect to the IMF seen in Fig. 1c for IMF $B_{y}$ is about $10^{\circ}$.

Figure 1f shows the GD model results for east-west IMF corresponding to the positions in Fig. 1c for an IMF $\left(B_{x}=-3.5, B_{y}=6.3, B_{z}=0\right) \mathrm{nT}$. Relative to the IMF, field vectors drape symmetrically around the object in a continuous manner, as expected for hydrodynamical flow around a blunt, axisymmetric object. This is in contrast with the IMP 8 data for the east-west IMF case.

Figure 1(i) gives the magnetic field vectors of the MHD model for east-west IMF corresponding to Fig. 1 $\mathrm{c}$ and $\mathrm{f}$. The figure shows a striking similarity between the field draping pattern of the MHD model vectors and the IMP 8 data, Fig. 1(c). The vector draping pattern in the model panel is rotated counter clockwise for positive IMF $B_{y}$ (away sector) as is observed in the data. The only differences between the MHD model and the observations seem to be the degree and the locations of the rotation. In both, the rotation of the observed field draping is more pronounced close to the magnetopause than in the outer region magnetosheath.

Figure 4 compares and quantifies the rotation of the draping patterns. In the figure, the MHD model results

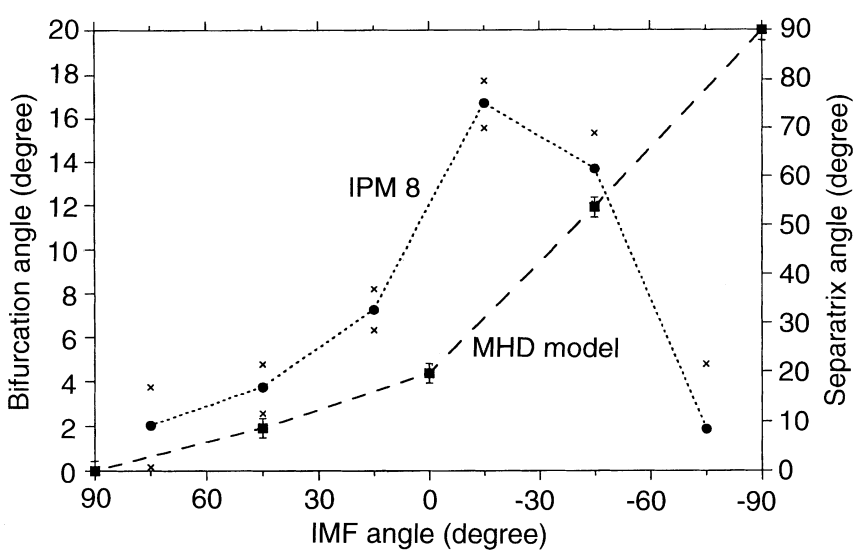

Fig. 4. Variation of the magnetopause rotation angle with IMF clock angle. Squares are the model values derived from Fedder et al. (1995) for comparison. The bars on the model values give the interval in which the separatrix angle changes within $2^{\circ}$ for different initial conditions. Circles give the rotation angle of the magnetopause as observed in IMP 8 measurements as a function of IMF angle. The crosses above and below the observations give the $1 \sigma$ deviations (from Kaymaz et al. 1992). Note that the definition of the rotation angle is different in the model than that calculated for IMP 8 data as described in the text and shown in Fig. 5. However, in both cases the rotation angle increases as the IMF changes its orientation from north to south. The biggest rotation is seen for equatorial IMFs with a nonzero southward component. (See text for more detailed discussion of this point) are taken from Fedder et al. (1995). The bars on the model results $\left(2^{\circ}\right)$ show the sensitivity of the model runs for different initial conditions. We see that the degree of rotation of the MHD inner magnetosheath draping pattern (Fedder et al., 1995) is comparable to the rotation of the observed field draping pattern (Kaymaz et al., 1992). The angle in the Fedder et al. (1995) study is the angle between the IMF direction and the separatrix line connecting the points separating the last closed field line from the first open field line. It is a true topological angle of the reconnection geometry in the MHD model. Figure 5a illustrates this geometry for the southward IMF case. The Kaymaz et al. (1992) angle is defined somewhat differently from the angle defined by Fedder et al. (1995). In Kaymaz et al. (1992), as shown in Fig. 5b, the angle is that between the IMF direction and the line which connects the bifurcation points where the tangential components of the field change direction. Because of the different definitions, whereas Fedder $e t$ al. (1995) observe the maximum rotation for pure southward IMF, Kaymaz et al. (1992) find the maximum for strong IMF $B_{y}$ with some southward IMF. However, Fig. 4 indicates that the rotation angle in both cases increases as the IMF clock angle changes from north to south. Thus, both the data and MHD model give essentially the same result. This shows that, despite the fact that both data sets result from two different features of the magnetic field configuration, they are caused by dayside reconnection geometry at the magnetopause.

\section{Summary}

This work attempts to show how well, on average GD and MHD models represent the magnetic field observations in the cross section of the magnetosheath $-30 R_{e}$ away from Earth for northward, southward and eastwest IMF orientations. For several decades, the GD model was the only magnetosheath model used to determine the field configuration and flow characteristics in the magnetosheath. Here we see that the GD model provides an accurate representation of the observed magnetosheath field draping in the cross section for northward IMFs. It is also a reasonable model for the outer magnetosheath for other IMFs. However, for the equatorial and southward cases, it fails to represent important aspects of magnetosheath field draping close to the magnetopause boundary where the magnetic forces become dominant and where magnetopause merging affects the field and flow. The GD model also predicts stronger fields than are observed on average because it does not take into account magnetic forces near the boundary nor the connection to the Earth's field at the boundary. The global MHD model, on the other hand, by nature includes these effects, and therefore it is expected to resemble more closely the real situation, especially at the magnetopause.

The results presented in this study illustrate the promise of MHD models for reproducing features similar to those observed. The magnetic field vector maps of the MHD model for the IMF cases examined 

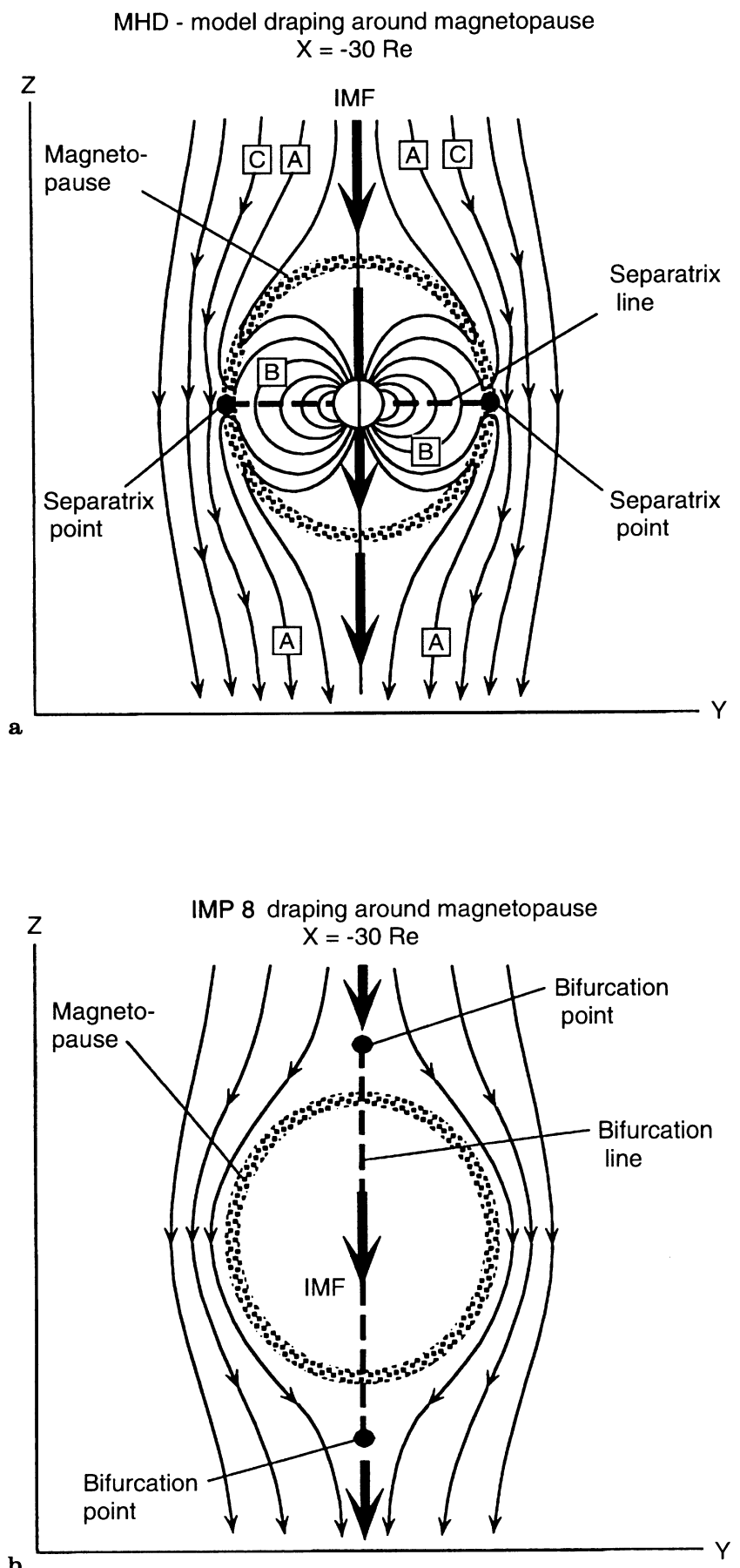

Fig. 5a, b. The definition of the rotation angles for a the MHD model (Fedder et al. (1995) and b IMP 8 data (Kaymaz et al. 1992). In the model, separatrix angle is the rotation angle of the magnetopause defined by the first open field line (a) and the last closed field line (b). In the figure, field lines and the separatrix line are shown projected onto the $y z$-plane. For IMP 8 data, the angle is the bifurcation angle where the tangential components of the fields change sign as the fields split to go around the body of the magnetosphere

indicate that the differences between the MHD model vectors and IMP 8 magnetic field observations are smaller close to the magnetopause where the GD model differs most. Qualitatively, both models produce a similar cross-sectional field geometry of the draped magnetosheath field at $-30 \mathrm{R}_{\mathrm{e}}$ for northward IMF, and quantitatively, both differ as much as $22 \%$ of the total concurrent IMF in field magnitudes from the observations. However, only the MHD model successfully reproduces the observed draping patterns for both east-west and southward IMFs near the boundary. Specifically, in contrast to the gas dynamic magnetosheath model, the fields in the MHD model drape around the tail magnetopause asymmetrically with respect to the IMF direction as observed. The twisting of the draping pattern increases as the IMF turns southward, also as observed. The greatest amount of rotation is seen in the inner magnetosheath when the IMF has a strong $B_{y}$ component with a southward component. Further, in regions of irregular fields in the southward case, the MHD model vectors are weaker than those in the gas dynamic model maps, and agree better with the observations.

The close qualitative agreements demonstrated between the MHD model and IMP 8 data are the primary results of this study because they show that interactions with the magnetosphere affect the draping of magnetosheath fields. Reconnection is evidently important in controlling the magnetosheath field geometry near the magnetopause boundary for all IMF orientations.

The GD magnetosheath model is expected to be inadequate in regions close to the magnetopause (Alksne, 1967) where magnetic forces become dominant and where the geomagnetic field and magnetosheath field interconnect, thereby violating the gas dynamic model assumption of an impenetrable magnetopause. The results of Kaymaz et al. (1992) indicated that magnetic reconnection particularly affects the magnetosheath field draping geometry as seen in cross section near the magnetopause for IMFs in the equatorial plane. Further, their results show that the magnetosheath field structure is different qualitatively and quantitatively for northward and southward IMFs. Proper representation of conditions at the magnetopause requires that the magnetic field and flow equations be solved together, with the inclusion of the IMF orientation and the coupling with magnetospheric fields.

Models of magnetosheath flow that take magnetic forces into account, MHD models, are not yet developed to the point of being easily used like the simpler GD model. The treatment of the dissipative terms in the MHD models requires a fine spatial grid over extended boundary regions. Even the most sophisticated MHD models have relatively poor spatial resolution and are costly to run. Nevertheless, as shown here, global MHD models are available which approach the observed magnetosheath fields in general appearance.

Recently, Kaymaz et al. (1995) used the same MHD model, the Fedder and Lyon global MHD model, for comparison with the observed magnetotail field geometry for different IMF orientations. They found that the model represents the magnetotail's gross characteristics fairly well as seen in cross section at $-30 \mathrm{R}_{\mathrm{e}}$. The MHD model captures the signatures of the solar-wind magnetotail coupling like skewing of the plasma sheet field lines, rotation of the tail current sheet, penetration of a 
fraction of IMF into the plasma sheet and the concentration of the penetrated fields on the tail flanks. Here the same MHD model is compared with the field data in the magnetosheath as well as with the GD model. We see that here too, the MHD model captures the particular observed features of the magnetosheath field, such as the rotation of the draping pattern in the presence of IMF $B_{\mathrm{y}}$, the degree of the rotation for different IMF directions, the generally smaller field magnitudes closer to the boundary for all IMFs, and the anomalous regions for southward IMFs. These comparisons serve to verify both the general validity of MHD models, in spite of their coarse resolution, and the general concept of the coupling to Earth's field through reconnection at the magnetopause.

\section{Conclusions}

1. For northward IMF, field draping patterns of the GD model and MHD model both agree with the observed draping pattern in the $y z$-plane. Both models predict stronger fields, in general, than observed by about $22 \%$ of the upstream field magnitude. This case shows the best agreement between the data and the models. The field patterns for the northward IMF are consistent with the idea that the magnetosphere is closed during northward IMF.

2. For southward IMF, there are anomalous regions of irregular, 'turbulent' structures where one might expect that reconnection of Earth's magnetic field lines with southward interplanetary fields would produce a small $B_{z}$ component close to the boundary. Whereas the GD model does not show anomalies where these patchy structures occur, the MHD model field shows weak fields and deviant directions at the appropriate locations.

3. For east-west IMFs, the GD model fails to show the rotation of the draping pattern of the magnetic field lines close to the magnetopause. The reason for this is the lack of magnetic interaction at the boundary between the field and flow. The MHD model, on the other hand, shows the asymmetric (rotated) draped field geometry, and further it predicts the degree of observed rotation. The absence of the rotation of the field draping in the GD model emphasizes the effects of magnetic tension resulting from reconnected field lines at the magnetopause, which are inherently part of MHD code.

In conclusion, this study shows that the magnetic coupling with the magnetosphere at the magnetopause creates a clear signature in IMP 8 observations near the magnetopause at $-30 \mathrm{R}_{\mathrm{e}}$. The magnetopasue merging origin of the signal is confirmed by comparing the observations with GD and MHD models

Acknowledgements. This work was supported by NSF (grant ATM 91-17484, University of California, Los Angeles). The author would like to thank J. Fedder, J. Lyon and S. Slinker for discussions about the MHD code and providing the MHD model data; to J. Spreiter and S. Stahara for providing the gas dynamic model code; and to G.L. Siscoe and J.G. Luhmann for useful conversations on the several aspects of this work.
The Editor in Chief thanks J. Fedder and J. Luhmann for their help in evaluating this paper.

\section{References}

Alksne, A. Y., The steady-state magnetic field in the transition region between the magnetosphere and the bow shock, Planet. Space. Sci., 15, 239, 1967.

Behannon, K. W., and D. H. Fairfield, Spatial variations of the magnetosheath magnetic field, Planet. Space Sci., 17, 1803, 1969.

Crooker, N. U., G. L. Siscoe, P. R. Mullen, C. T. Russell, and E. J. Smith, magnetic field compression at the dayside, J. Geophys. Res., 87, 10407, 1982.

Fairfield, D. H., The ordered magnetic field of the magnetosheath, J. Geophys. Res., 72, 1967.

Fairfield, D. H., Magnetic fields of the magnetosheath, Rev. Geophys., 14, 1, 1976.

Fedder, J. A., and J. Lyon, The Earth's magnetosphere is $165 \mathrm{Re}$ long: or self consistent currents, convection, magnetospheric structure and processes for northward IMF, J. Geophys. Res., 100, 3623, 1995.

Fedder, J. A., J. G. Lyon, S. P. Slinker and C. M. Mobarry, Topological Structure of the magnetotail as a function of IMF direction, J. Geophys. Res., 100, 3613, 1995.

Gonzales, W. D. and F. S. Mozer, A quantitative model for potential resulting from reconnection with an arbitrary interplanetary magnetic field, J. Geophys. Res., 79, 4186, 1974.

Gurnett, D. A., and L. A. Frank, Observed relationships between electric fields and auroral particle precipitation, J. Geophys. Res., 78, 145, 1973.

Kaymaz, Z., G. L. Siscoe, and J. G. Luhmann, IMF draping around the geotail: IMP 8 observations, Geophys. Res. Lett., 19, 8, 829, 1992.

Kaymaz, Z., G. L. Siscoe, J. G. Luhmann, R. P. Lepping and C. T. Russell, Interplanetary field control of magnetotail field geometry: IMP 8 observations, J. Geophys. Res., 99, 11113, 1994.

Kaymaz, Z., G. L. Siscoe, J. G. Luhmann, J. A. Fedder, and J. G. Lyon, Interplanetary field control of magnetotail field: IMP 8 data and MHD model compared, J. Geophys. Res., 100, 17163, 1995.

Luhmann, J.G., R.J. Walker, C.T. Russell, N.U. Crooker, J.R. Spreiter, and S. S. Stahara, Patterns of potential magnetic field merging sites on the dayside magnetopause, J. Geophys. Res., 89, $1739,1984$.

Luhmann, J. G., R. J. Walker, C. T. Russell, J. R. Spreiter, S. S. Stahara, and D. J. Williams, Mapping the magnetosheath field between the magnetopause and bow shock: implications for magnetospheric particle leakage, J. geophys. Res., 89, 6829, 1986.

Petrinec, S. M., C. T. Russell, External and internal influences on the size of the dayside terrestrial magnetosphere, Geophys. Res. Lett., 20, 339, 1993.

Reiff, P. H., J. G. Luhmann, Solar wind control of the polar cap voltage, in Solar wind magnetosphere coupling, Eds. Y. Kamide and J. A. Slavin, 453, 1986.

Siscoe, G. L., and E. R. Sanchez, IMP 8 magnetotail boundary crossings: a test of the MHD models for an open magnetosphere, J. Geophys. Res., 95, 20771, 1990.

Sanchez, E. R. and G. L. Siscoe, An MHD model for complete open magnetotail boundary, J. Geophys. Res., 92, 7405, 1987.

Slavin, J. A., R. E. Holzer, J. R. Spreiter, S. S. Stahara, and D. S. Chausse, Solar Wind flow about the terrestrial planets, 2: comparison with gas dynamic theory and implications for solar planetary interactions, J. Geophys. Res., 88, 19, 1983.

Song, P., C. T. Russell, and M. F. Thomsen, Slow mode transition in the frontside magnetosheath, J. Geophys. Res., 97, 8295, 1992.

Spreiter, J. R., and A. Y. Alksne, Plasma flow around the magnetosphere, in Magnetospheric physics, Eds. by D. J. Williams and G.D. Mead, AGU, Washington, D.C., 11, 1969. 
Spreiter, J. R., and A. Y. Alksne, Solar wind flow past objects in the solar system, Ann.Rev.df Fluid Mech., 2, 513, 1970.

Spreiter, J. R., and S. S. Stahara, A new predictive model for determining solar wind terrestrial planet interactions, J. Geophys. Res., 85, 6769, 1980.

Spreiter, J. R., A. L. Summers, and A. Y. Alksne, Hydrodynamic flow around the magnetosphere, Planet. Space Sci., 14, 223, 1966.

Spreiter, J. R., A. Y. Alksne, and A. L. Summers, External aerodynamic of the magnetosphere, in Physics of the magnetosphere, Eds. R. L. Carovillano, J. F. McClay, and, H. R. Radoski, D. Reid, Dordrecht, Holland, 301, 1968.
Stahara, S. S., D. Klenke, B. C., Trudinger, and J. R. Spreiter, Applications of advanced computational procedures for modeling solar wind interactions with Venus-theory and computer code, NASA Contract Rep., Near TR 202, 1979.

Zhang, X. X., P. Song, S. S. Stahara, J. R. Spreiter, C. T. Russell, and G. Le, Large-scale structures in the magnetosheath: Exgenous or endogenous in origin?, Geophys. Res. Lett., 23, 105, 1996.

Zwan, B. J. and R. A. Wolf, Depletion of solar wind plasma near a planetary boundary, J. Geophys. Res., 81, 1636, 1976. 